\title{
More properties of the proportional fractional integrals and derivatives of a function with respect to another function
}

\author{
Fahd Jarad ${ }^{1}$, Thabet Abdeljawad ${ }^{2,3,4^{*}}$ (D), Saima Rashid ${ }^{5}$ and Zakia Hammouch ${ }^{6}$
}

"Correspondence:

tabdeljawad@psu.edu.sa

${ }^{2}$ Department of Mathematics and

General Sciences, Prince Sultan

University, Riyadh, Saudi Arabia

${ }^{3}$ Department of Medical Research,

China Medical University, Taichung, Taiwan

Full list of author information is

available at the end of the article

\begin{abstract}
In this article, we present some new properties of the fractional proportional derivatives of a function with respect to a certain function. We use a modified Laplace transform to find the relation between the derivatives in the Riemann-Liouville setting and the one in Caputo. In addition, we provide an integration by parts formulas related to the considered operators.
\end{abstract}

Keywords: General proportional integrals; General proportional derivatives; General Caputo proportional derivative

\section{Introduction}

For the last 30 years or more, some scientists have shown a great deal of interest in the field of fractional calculus which addresses the derivatives and integrals with any order. As a matter of fact, this interest has sprung out by the dint of the substantial results obtained when these scientists used the tools in this calculus in order to study some models from the real world [1-6].

One of the virtues of the classical fractional calculus is that there are a variety of derivatives and integrals. Nevertheless, there has always been a need to develop this calculus more and discover some new derivatives for the sake of better understanding the universe. Some of the newly proposed fractional operators contain nonsingular kernels [7-14]. In 2011 , Katugampola in $[15,16]$ proposed what he called generalized fractional operators for the purpose of combining the Riemann-Liouville and Hadamard fractional operators. The generalized derivatives were modified so that they cover the Caputo and the CaputoHadamard fractional derivatives [17]. In other respects, local derivatives permit differentiation and integration of noninteger order. In $[18,19]$, the authors introduced what they called conformable derivative. It should be noted that the nonlocal fractional versions of the conformable operators were discussed in $[15,16,20]$. However, more generalized forms of these operators were discussed in [21].

The main disadvantage of the conformable derivative is that contrary to the other differential operators it does not produce the function itself once the order is 0 . To overcome this, the authors in $[22,23]$ proposed a modification of the conformable derivative so that

(c) The Author(s) 2020. This article is licensed under a Creative Commons Attribution 4.0 International License, which permits use, sharing, adaptation, distribution and reproduction in any medium or format, as long as you give appropriate credit to the original author(s) and the source, provide a link to the Creative Commons licence, and indicate if changes were made. The images or other third party material in this article are included in the article's Creative Commons licence, unless indicated otherwise in a credit line to the material. If material is not included in the article's Creative Commons licence and your intended use is not permitted by statutory regulation or exceeds the permitted use, you will need to obtain permission directly from the copyright holder. To view a copy of this licence, visit http://creativecommons.org/licenses/by/4.0/. 
if the order of this derivative tends to 0 it gives the function itself and if the order tends to 1 it gives the first-order derivative of the function. Later, the authors in [24] presented a new type of fractional operators generated from the above-mentioned modified conformable derivatives. In addition, more generalized forms of these fractional operators were put forward in [25], and it turned out that some of these operators coincided with some operators mentioned before in [26-28].

In this work, orientated by the above-mentioned works, we continue our study on the proportional fractional derivatives and integrals of a function with respect to another function discovered in [25]. We present the effect of the fractional integral operators on the differential operators and vice versa. In addition, we present the relation between the fractional proportional derivatives in Riemann-Liouville and Caputo settings using a modified Laplace transform.

The article is organized as follows: Sect. 2 is devoted to some essential definitions for fractional proportional derivative and integrals and their generalizations. In Sect. 3, we apply the proportional fractional integrals on fractional derivatives, discuss the Laplace transforms for the generalized fractional integrals and derivatives, and give some examples. In Sect. 4, we present an integration by parts formula, and we conclude our work in Sect. 5.

\section{Essential preliminaries}

In this section, we present fundamental definitions, lemmas, theorems, and corollaries needed for our findings in this article.

\subsection{The proportional derivatives}

Definition 2.1 (Modified conformable derivatives; $[22,23]$ ) For $\varrho \in[0,1]$, let the functions $\varkappa_{0}, \varkappa_{1}:[0,1] \times \mathbb{R} \rightarrow[0, \infty)$ be continuous such that for all $t \in \mathbb{R}$ we have

$$
\lim _{\varrho \rightarrow 0^{+}} \varkappa_{1}(\varrho, t)=1, \quad \lim _{\varrho \rightarrow 0^{+}} \varkappa_{0}(\varrho, t)=0, \quad \lim _{\varrho \rightarrow 1^{-}} \varkappa_{1}(\varrho, t)=0, \quad \lim _{\varrho \rightarrow 1^{-}} \varkappa_{0}(\varrho, t)=1,
$$

and $\varkappa_{1}(\varrho, t) \neq 0, \varrho \in[0,1), \varkappa_{0}(\varrho, t) \neq 0, \varrho \in(0,1]$. Then the modified conformable differential operator of order $\varrho$ is defined by

$$
D^{\varrho} f(t)=\varkappa_{1}(\varrho, t) f(t)+\varkappa_{0}(\varrho, t) f^{\prime}(t) .
$$

The derivative given in (2.1) is called a proportional derivative. For more details about the control theory of the proportional derivatives and their component functions $\varkappa_{0}$ and $\varkappa_{1}$, we refer the reader to $[22,23]$.

For the restricted case when $\varkappa_{1}(\varrho, t)=1-\varrho$ and $\varkappa_{0}(\varrho, t)=\varrho$, the proportional derivative and its integral respectively read

$$
D^{\varrho} f(t)=(1-\varrho) f(t)+\varrho f^{\prime}(t)
$$

and

$$
{ }_{a} I^{1, \varrho} f(t)=\frac{1}{\varrho} \int_{a}^{t} e^{\frac{\varrho-1}{\varrho}(t-s)} f(s) d s,
$$

where ${ }_{a}{ }^{0, \varrho} f(t)=f(t)$. 
The $n$th order proportional integral has the form

$$
\left({ }_{a} I^{n, \varrho} f\right)(t)=\frac{1}{\varrho^{n} \Gamma(n)} \int_{a}^{t} e^{\frac{\varrho-1}{\varrho}(t-\tau)}(t-\tau)^{n-1} f(\tau) d \tau .
$$

Definition 2.2 ([24]) For $\varrho>0$ and $\alpha \in \mathbb{C}, \operatorname{Re}(\alpha)>0$, the left and right proportional fractional integrals of $f$ are respectively defined as

$$
\left({ }_{a} I^{\alpha, \varrho} f\right)(t)=\frac{1}{\varrho^{\alpha} \Gamma(\alpha)} \int_{a}^{t} e^{\frac{\varrho-1}{\varrho}(t-\tau)}(t-\tau)^{\alpha-1} f(\tau) d \tau
$$

and

$$
\left(I_{b}^{\alpha, \varrho} f\right)(t)=\frac{1}{\varrho^{\alpha} \Gamma(\alpha)} \int_{t}^{b} e^{\frac{\varrho-1}{\varrho}(\tau-t)}(\tau-t)^{\alpha-1} f(\tau) d \tau .
$$

Definition 2.3 ([24]) For $\varrho>0$ and $\alpha \in \mathbb{C}, \operatorname{Re}(\alpha) \geq 0$, the left and right proportional derivatives of $f$ are respectively given as

$$
\begin{aligned}
\left({ }_{a} D^{\alpha, \varrho} f\right)(t) & =D^{n, \varrho}{ }_{a} I^{n-\alpha, \varrho} f(t) \\
& =\frac{D_{t}^{n, \varrho}}{\varrho^{n-\alpha} \Gamma(n-\alpha)} \int_{a}^{t} e^{\frac{\varrho-1}{\varrho}(t-\tau)}(t-\tau)^{n-\alpha-1} f(\tau) d \tau
\end{aligned}
$$

and

$$
\begin{aligned}
\left(D_{b}^{\alpha, \varrho} f\right)(t) & ={ }_{\ominus} D^{n, \varrho} I_{b}^{n-\alpha, \varrho} f(t) \\
& =\frac{{ }_{\ominus} D_{t}^{n, \varrho}}{\varrho^{n-\alpha} \Gamma(n-\alpha)} \int_{t}^{b} e^{\frac{\varrho-1}{\varrho}(\tau-t)}(\tau-t)^{n-\alpha-1} f(\tau) d \tau,
\end{aligned}
$$

where $n=[\operatorname{Re}(\alpha)]+1$,

$$
D^{n, \varrho}=\underbrace{D^{\varrho} D^{\varrho} \ldots D^{\varrho}}_{n \text { times }}
$$

and

$$
\left({ }_{\ominus} D^{\varrho} f\right)(t):=(1-\varrho) f(t)-\varrho f^{\prime}(t), \quad{ }_{\ominus} D^{n, \varrho}=\underbrace{{ }_{\ominus} D_{\ominus}^{\varrho} D^{\varrho} \ldots{ } D^{\varrho}}_{n \text { times }} .
$$

\subsection{The fractional proportional derivative of a function with respect to another function}

Definition 2.4 (The proportional derivative of a function with respect to another function; [25]) For $\varrho \in[0,1]$, let the functions $\varkappa_{0}, \varkappa_{1}:[0,1] \times \mathbb{R} \rightarrow[0, \infty)$ be continuous such that for all $t \in \mathbb{R}$ we have

$$
\lim _{\varrho \rightarrow 0^{+}} \varkappa_{1}(\varrho, t)=1, \quad \lim _{\varrho \rightarrow 0^{+}} \varkappa_{0}(\varrho, t)=0, \quad \lim _{\varrho \rightarrow 1^{-}} \varkappa_{1}(\varrho, t)=0, \quad \lim _{\varrho \rightarrow 1^{-}} \varkappa_{0}(\varrho, t)=1,
$$

and $\varkappa_{1}(\varrho, t) \neq 0, \varrho \in[0,1), \varkappa_{0}(\varrho, t) \neq 0, \varrho \in(0,1]$. Let also $g(t)$ be a strictly increasing continuous function. Then the proportional differential operator of order $\varrho$ of $f$ with respect 
to $g$ is defined by

$$
D^{\varrho, g} f(t)=\varkappa_{1}(\varrho, t) f(t)+\varkappa_{0}(\varrho, t) \frac{f^{\prime}(t)}{g^{\prime}(t)}
$$

As in the previous subsection, for the restriction $\varkappa_{1}(\varrho, t)=1-\varrho$ and $\varkappa_{0}(\varrho, t)=\varrho,(2.1)$ becomes

$$
D^{\varrho, g} f(t)=(1-\varrho) f(t)+\varrho \frac{f^{\prime}(t)}{g^{\prime}(t)} .
$$

The corresponding integral of (2.10) of order $n$ [25]

$$
\left({ }_{a} I^{n, \varrho, g} f\right)(t)=\frac{1}{\varrho^{n} \Gamma(n)} \int_{a}^{t} e^{\frac{\varrho-1}{\varrho}(g(t)-g(\tau))}(g(t)-g(\tau))^{n-1} f(\tau) g^{\prime}(\tau) d \tau .
$$

The left and right proportional integrals in their general forms are given as follows.

Definition 2.5 ([25]) For $\varrho \in(0,1], \alpha \in \mathbb{C}, \operatorname{Re}(\alpha)>0$, we define the left and right fractional integrals of $f$ with respect to $g$ by

$$
\left({ }_{a} I^{\alpha, \varrho, g} f\right)(t)=\frac{1}{\varrho^{\alpha} \Gamma(\alpha)} \int_{a}^{t} e^{\frac{\varrho-1}{\varrho}(g(t)-g(\tau))}(g(t)-g(\tau))^{\alpha-1} f(\tau) g^{\prime}(\tau) d \tau .
$$

The right fractional proportional integral ending at $b$, however, can be defined by

$$
\begin{aligned}
& \left(I_{b}^{\alpha, \varrho, g} f\right)(t)=\frac{1}{\varrho^{\alpha} \Gamma(\alpha)} \int_{t}^{b} e^{\frac{\varrho-1}{\varrho}(g(\tau)-g(t))}(g(\tau)-g(t))^{\alpha-1} f(\tau) g^{\prime}(\tau) d \tau, \\
& \left({ }_{a} I^{\alpha, \varrho, g} f\right)(t)=\frac{1}{\varrho^{\alpha} \Gamma(\alpha)} \int_{a}^{t} e^{\frac{\varrho-1}{\varrho}(g(t)-g(\tau))}(g(t)-g(\tau))^{\alpha-1} f(\tau) g^{\prime}(\tau) d \tau,
\end{aligned}
$$

and

$$
\left(I_{b}^{\alpha, \varrho, g} f\right)(t)=\frac{1}{\varrho^{\alpha} \Gamma(\alpha)} \int_{t}^{b} e^{\frac{\varrho-1}{\varrho}(g(\tau)-g(t))}(g(\tau)-g(t))^{\alpha-1} f(\tau) g^{\prime}(\tau) d \tau .
$$

Definition 2.6 ([25]) For $\varrho>0, \alpha \in \mathbb{C}, \operatorname{Re}(\alpha) \geq 0$, and $g \in C[a, b]$, where $g^{\prime}(t)>0$, we define the left fractional derivative of $f$ with respect to $g$ as

$$
\begin{aligned}
\left({ }_{a} D^{\alpha, \varrho, g} f\right)(t)= & D^{n, \varrho, g}{ }_{a} I^{n-\alpha, \varrho, g} f(t) \\
= & \frac{D_{t}^{n, \varrho, g}}{\varrho^{n-\alpha} \Gamma(n-\alpha)} \int_{a}^{t} e^{\frac{\varrho-1}{\varrho}(g(t)-g(\tau))}(g(t)-g(\tau))^{n-\alpha-1} \\
& \times f(\tau) g^{\prime}(\tau) d \tau
\end{aligned}
$$

and the right fractional derivative of $f$ with respect to $g$ as

$$
\begin{aligned}
\left(D_{b}^{\alpha, \varrho, g} f\right)(t) & ={ }_{\ominus} D^{n, \varrho, g} I_{b}^{n-\alpha, \varrho, g} f(t) \\
& =\frac{{ }_{\ominus} D_{t}^{n, \varrho, g}}{\varrho^{n-\alpha} \Gamma(n-\alpha)} \int_{t}^{b} e^{\frac{\varrho-1}{\varrho}(g(\tau)-g(t))}(g(\tau)-g(t))^{n-\alpha-1}
\end{aligned}
$$




$$
\times f(\tau) g^{\prime}(\tau) d \tau
$$

where $n=[\operatorname{Re}(\alpha)]+1$,

$$
D^{n, \varrho, g}=\underbrace{D^{\varrho, g} D^{\varrho, g} \ldots D^{\varrho, g}}_{n \text { times }}
$$

and

$$
\left({ }_{\ominus} D^{\varrho, g} f\right)(t):=(1-\varrho) f(t)-\varrho \frac{f^{\prime}(t)}{g^{\prime}(t)}, \quad{ }_{\ominus} D^{n, \varrho, g}=\underbrace{{ }_{\ominus} D^{\varrho, g}{ }_{\ominus} D^{\varrho, g} \ldots{ }_{\ominus} D^{\varrho, g}}_{n \text { times }} .
$$

Proposition 2.1 ([25]) Let $\alpha, \beta \in \mathbb{C}$ be such that $\operatorname{Re}(\alpha) \geq 0$ and $\operatorname{Re}(\beta)>0$. Then, for any $\varrho>0$, we have

1. $\left({ }_{a} I^{\alpha, \varrho, g} e^{\frac{\varrho-1}{\varrho} g(x)}(g(x)-g(a))^{\beta-1}\right)(t)=\frac{\Gamma(\beta)}{\Gamma(\beta+\alpha) \varrho^{\alpha}} e^{\frac{\varrho-1}{\varrho} g(t)}(g(t)-g(a))^{\alpha+\beta-1}$, $\operatorname{Re}(\alpha)>0$.

2. $\left(I_{b}^{\alpha, \varrho, g} e^{-\frac{\varrho-1}{\varrho} g(x)}(g(b)-g(x))^{\beta-1}\right)(t)=\frac{\Gamma(\beta)}{\Gamma(\beta+\alpha) \varrho^{\alpha}} e^{-\frac{\varrho-1}{\varrho} g(t)}(g(b)-g(t))^{\alpha+\beta-1}$, $\operatorname{Re}(\alpha)>0$.

3. $\left({ }_{a} D^{\alpha, \varrho} e^{\frac{\varrho-1}{\varrho} g(x)}(g(x)-g(a))^{\beta-1}\right)(t)=\frac{\varrho^{\alpha} \Gamma(\beta)}{\Gamma(\beta-\alpha)} e^{\frac{\varrho-1}{\varrho} g(t)}(g(t)-g(a))^{\beta-1-\alpha}$, $\operatorname{Re}(\alpha) \geq 0$

4. $\left(D_{b}^{\alpha, \varrho, g} e^{-\frac{\varrho-1}{\varrho} g(x)}(g(b)-g(x))^{\beta-1}\right)(t)=\frac{\varrho^{\alpha} \Gamma(\beta)}{\Gamma(\beta-\alpha)} e^{-\frac{\varrho-1}{\varrho} g(t)}(g(b)-g(t))^{\beta-1-\alpha}$, $\operatorname{Re}(\alpha) \geq 0$

Theorem 2.1 ([25]) If $\varrho \in(0,1], \operatorname{Re}(\alpha)>0$, and $\operatorname{Re}(\beta)>0$. Then, for $f$ is continuous and defined for $t \geq a$, we have

$$
\begin{aligned}
& { }_{a} I^{\alpha, \varrho, g}\left({ }_{a} I^{\beta, \varrho, g} f\right)(t)={ }_{a} I^{\beta, \varrho, g}\left({ }_{a} I^{\alpha, \varrho, g} f\right)(t)=\left({ }_{a} I^{\alpha+\beta, \varrho, g} f\right)(t), \\
& I_{b}^{\alpha, \varrho, g}\left(I_{b}^{\beta, \varrho, g} f\right)(t)=I_{b}^{\beta, \varrho, g}\left(I_{b}^{\alpha, \varrho, g} f\right)(t)=\left(I_{b}^{\alpha+\beta, \varrho, g} f\right)(t) .
\end{aligned}
$$

Theorem $2.2([25])$ Let $0 \leq m<[\operatorname{Re}(\alpha)]+1$ and $f$ be integrable in each interval $[a, t], t>a$. Then

$$
D^{m, \varrho, g}\left({ }_{a} I^{\alpha, \varrho, g} f\right)(t)=\left({ }_{a} I^{\alpha-m, \varrho, g} f\right)(t)
$$

and

$$
{ }_{\ominus} D^{m, \varrho, g}\left(I_{b}^{\alpha, \varrho, g} f\right)(t)=\left(I_{b}^{\alpha-m, \varrho, g} f\right)(t)
$$

Corollary $2.1([25])$ Let $0<\operatorname{Re}(\beta)<\operatorname{Re}(\alpha)$ and $m-1<\operatorname{Re}(\beta) \leq m$. Then we have

$$
{ }_{a} D^{\beta, \varrho, g}{ }_{a} I^{\alpha, \varrho, g} f(t)={ }_{a} I^{\alpha-\beta, \varrho, g} f(t)
$$


and

$$
D_{b}^{\beta, \varrho, g} I_{b}^{\alpha, \varrho, g} f(t)=I_{b}^{\alpha-\beta, \varrho, g} f(t) .
$$

Corollary 2.2 ([25]) Let $f$ be integrable on $t \geq a$ and $\operatorname{Re}[\alpha]>0, \varrho \in(0,1], n=[\operatorname{Re}(\alpha)]+1$. Then we have

$$
{ }_{a} D^{\alpha, \varrho, g}{ }_{a}^{\alpha, \varrho, g} f(t)=f(t)
$$

and

$$
D_{b}^{\alpha, \varrho, g} I_{b}^{\alpha, \varrho, g} f(t)=f(t)
$$

The generalized Caputo proportional fractional derivatives are given as follows.

Definition 2.7 ([25]) For $\varrho \in(0,1]$ and $\alpha \in \mathbb{C}$ with $\operatorname{Re}(\alpha) \geq 0$, we define the left derivative of Caputo type starting at $a$ by

$$
\begin{aligned}
\left({ }_{a}^{C} D^{\alpha, \varrho, g} f\right)(t)= & { }_{a} I^{n-\alpha, \varrho, g}\left(D^{n, \varrho, g} f\right)(t) \\
= & \frac{1}{\varrho^{n-\alpha} \Gamma(n-\alpha)} \int_{a}^{t} e^{\frac{\varrho-1}{\varrho}(g(t)-g(\tau))}(g(t)-g(\tau))^{n-\alpha-1} \\
& \times\left(D^{n, \varrho, g} f\right)(\tau) g^{\prime}(\tau) d \tau .
\end{aligned}
$$

The right derivative of Caputo type ending at $b$ is defined by

$$
\begin{aligned}
\left({ }^{C} D_{b}^{\alpha, \varrho} f\right)(t)= & I_{b}^{n-\alpha, \varrho, g}\left({ }_{\ominus} D^{n, \varrho, g} f\right)(t) \\
= & \frac{1}{\varrho^{n-\alpha} \Gamma(n-\alpha)} \int_{t}^{b} e^{\frac{\varrho-1}{\varrho}(g(\tau)-g(t))}(g(\tau)-g(t))^{n-\alpha-1} \\
& \times\left({ }_{\ominus} D^{n, \varrho, g} f\right)(\tau) g^{\prime}(\tau) d \tau,
\end{aligned}
$$

where $n=[\operatorname{Re}(\alpha)]+1$.

Proposition $2.2([25])$ Let $\alpha, \beta \in \mathbb{C}$ be such that $\operatorname{Re}(\alpha)>0$ and $\operatorname{Re}(\beta)>0$. Then, for any $\varrho>0$ and $n=[\operatorname{Re}(\alpha)]+1$, we have

1. $\left({ }_{a}^{C} D^{\alpha, \varrho, g} e^{\frac{\varrho-1}{\varrho} g(x)}(g(x)-g(a))^{\beta-1}\right)(t)=\frac{\varrho^{\alpha} \Gamma(\beta)}{\Gamma(\beta-\alpha)} e^{\frac{\varrho-1}{\varrho} g(t)}(g(t)-g(a))^{\beta-1-\alpha}$,

$\operatorname{Re}(\beta)>n$

2. $\left({ }^{C} D_{b}^{\alpha, \varrho, g} e^{-\frac{\varrho-1}{\varrho} g(x)}(g(b)-g(x))^{\beta-1}\right)(t)=\frac{\varrho^{\alpha} \Gamma(\beta)}{\Gamma(\beta-\alpha)} e^{\frac{\varrho-1}{\varrho}(g(b)-g(x))}(g(b)-g(t))^{\beta-1-\alpha}$, $\operatorname{Re}(\beta)>n$.

For $k=0,1, \ldots, n-1$, we have

$$
\left({ }_{a}^{C} D^{\alpha, \varrho, g} e^{\frac{\varrho-1}{\varrho} g(x)}\left(g(x)-g(a)^{k}\right)(t)=0 \quad \text { and } \quad\left({ }^{C} D_{b}^{\alpha, \varrho, g} e^{-\frac{\varrho-1}{\varrho} g(x)}(g(b)-g(x))^{k}\right)(t)=0 .\right.
$$

In particular, $\left({ }_{a}^{C} D^{\alpha, \varrho} e^{\frac{\varrho-1}{\varrho} g(x)}\right)(t)=0$ and $\left({ }^{C} D_{b}^{\alpha, \varrho} e^{-\frac{\varrho-1}{\varrho} g(x)}\right)(t)=0$. 


\subsection{The g-Laplace transforms}

The g-Laplace transform was proposed by Jarad et al. [21].

Definition 2.8 Let $f, g:[a, \infty) \rightarrow \mathbb{R}$ be real-valued functions such that $g(t)$ is continuous and $g^{\prime}(t)>0$ on $[a, \infty)$. The generalized Laplace transform of $f$ is defined by

$$
\mathcal{L}_{g}\{f(t)\}(s)=\int_{a}^{\infty} e^{-s(g(t)-g(a))} f(t) g^{\prime}(t) d t
$$

for all values of $s$, the integral is valid.

The generalized Laplace transforms of some elementary functions were given in the following lemma.

Lemma $2.1([21])$

1. $\mathcal{L}_{g}\{1\}(s)=\frac{1}{s}, \quad s>0$.

2. $\mathcal{L}_{g}\left\{(g(t)-g(a))^{\beta}\right\}(s)=\frac{\Gamma(\beta+1)}{s^{\beta+1}}, \quad \Re(\beta)>0, s>0$.

3. $\mathcal{L}_{g}\left\{e^{\lambda(g(t)-g(a))}\right\}(s)=\frac{1}{s-\lambda}, \quad s>\lambda$.

4. $\mathcal{L}_{g}\left\{e^{\lambda(g(t)-g(a))} f(t)\right\}(s)=\mathcal{L}_{g}\{f\}(s-\lambda)$.

The generalized Laplace transform of the derivative of $f$ with respect to $g$ is given as follows.

Theorem 2.3 ([21]) Let the function $f(t) \in C_{g}[a, T]$ and of $g(t)$-exponential order such that $f^{[1]}(t)$ is piecewise continuous over every finite interval $[a, T]$. Then the generalized Laplace transform of $f^{[1]}(t)=\frac{f^{\prime}}{\bar{g}^{\prime}}(t)$ exists and

$$
\mathcal{L}_{g}\left\{f^{[1]}(t)\right\}(s)=s \mathcal{L}_{g}\{f(t)\}(s)-f(a) .
$$

The generalized convolution integral is defined as follows.

Definition 2.9 ([21]) Let $f$ and $h$ be two functions which are piecewise continuous at each interval $[0, T]$ and of g-exponential order. The generalized convolution of $f$ and $h$ is defined by

$$
\left(f *_{g} h\right)(t)=\int_{a}^{t} f(\tau) h\left(g^{-1}(g(t)+g(a)-g(\tau))\right) g^{\prime}(\tau) d \tau .
$$

The generalized convolution of two functions is commutative.

Lemma 2.2 ([21]) Let $f$ and $h$ be two functions which are piecewise continuous at each interval $[a, T]$ and of exponential order. Then

$$
f *_{g} h=h *_{g} f .
$$


Theorem 2.4 ([21]) Let $f$ and $h$ be two functions which are piecewise continuous at each interval $[a, T]$ and of g-exponential order. Then

$$
\mathcal{L}_{g}\left\{f *_{g} h\right\}(s)=\mathcal{L}_{g}\{f\}(s) \mathcal{L}_{g}\{h\}(s) .
$$

\section{The main results}

Lemma 3.1 For $\alpha>1, \varrho \in(0,1]$, we have

$$
\left({ }_{a} I^{\alpha, \varrho, g} D^{1, \varrho, g} f\right)(t)=\left(D^{1, \varrho, g}{ }_{a} I^{\alpha, \varrho, g} f\right)(t)-\frac{(g(t)-g(a))^{\alpha-1} e^{\frac{\varrho-1}{\varrho}(g(t)-g(a))}}{\Gamma(\alpha) \varrho^{\alpha-1}} f(a)
$$

and

$$
\left(I_{b}^{\alpha, \varrho, g}{ }_{\ominus} D^{1, \varrho, g} f\right)(t)=\left({ }_{\ominus} D^{1, \varrho, g} I_{b}^{\alpha, \varrho, g} f\right)(t)-\frac{(g(b)-g(t))^{\alpha-1} e^{\frac{\varrho-1}{\varrho}(g(b)-g(t))}}{\Gamma(\alpha) \varrho^{\alpha-1}} f(b) .
$$

Proof Using the Leibniz rule, we can prove that

$$
\frac{\alpha-1}{\varrho^{\alpha-1} \Gamma(\alpha)} \int_{a}^{t} e^{\frac{\varrho-1}{\varrho}(g(t)-g(\tau))}(g(t)-g(\tau))^{\alpha-2} f(\tau) g^{\prime}(\tau) d \tau=D^{1, \varrho, g} I_{a}^{\alpha, \varrho, g} f(t) .
$$

Now, by using Definition 2.9,

$$
\begin{aligned}
\left({ }_{a}{ }^{\alpha, \varrho, g} D^{1, \varrho, g} f\right)(t)= & (1-\varrho) I^{\alpha, \varrho, g} f(t) \\
& +\frac{\alpha-1}{\varrho^{\alpha-1} \Gamma(\alpha)} \int_{a}^{t} e^{\frac{\varrho-1}{\varrho}(g(t)-g(\tau))}(g(t)-g(\tau))^{\alpha-1} f^{\prime}(\tau) d \tau .
\end{aligned}
$$

Now, using the integration by parts formula, we obtain

$$
\begin{aligned}
& \left(a^{\alpha, \varrho, g} D^{1, \varrho, g} f\right)(t) \\
& =(1-\varrho) I^{\alpha, \varrho, g} f(t)-\frac{(g(t)-g(a))^{\alpha-1} e^{\frac{\varrho-1}{\varrho}(g(t)-g(a))}}{\Gamma(\alpha) \varrho^{\alpha-1}} f(a) \\
& \quad+(\varrho-1) I^{\alpha, \varrho, g} f(t)+\frac{\alpha-1}{\varrho^{\alpha} \Gamma(\alpha)} \int_{a}^{t} e^{\frac{\varrho-1}{\varrho}(g(t)-g(\tau))}(g(t)-g(\tau))^{\alpha-2} f(\tau) g^{\prime}(\tau) d \tau \\
& =D^{1, \varrho, g} I_{a}^{\alpha, \varrho, g} f(t)-\frac{(g(t)-g(a))^{\alpha-1} e^{\frac{\varrho-1}{\varrho}(g(t)-g(a))}}{\Gamma(\alpha) \varrho^{\alpha-1}} f(a) .
\end{aligned}
$$

(3.2) can be proved similarly.

We can generalize Lemma 3.1 as follows.

Corollary 3.1 For $\alpha>m, \varrho \in(0,1]$, and $m$ is a positive integer, we have

$$
\begin{aligned}
\left({ }_{a}{ }^{\alpha, \varrho, g} D^{m, \varrho, g} f\right)(t)= & \left(D^{m, \varrho, g}{ }_{a} I^{\alpha, \varrho, g} f\right)(t) \\
& -\sum_{k=0}^{m-1} \frac{(g(t)-g(a))^{\alpha-m+k} e^{\frac{\varrho-1}{\varrho}(g(t)-g(a))}}{\Gamma(\alpha+k-m+1) \varrho^{\alpha-m+k}}\left(D^{k, \varrho, g} f\right)(a),
\end{aligned}
$$




$$
\begin{aligned}
\left(I_{b}^{\alpha, \varrho, g}{ }_{\ominus} D^{m, \varrho, g} f\right)(t)= & \left({ }_{\ominus} D^{m, \varrho, g} I_{b}^{\alpha, \varrho, g} f\right)(t) \\
& -\sum_{k=0}^{m-1} \frac{(-1)^{k}(g(t)-g(a))^{\alpha-m+k} e^{\frac{\varrho-1}{\varrho}(g(t)-g(a))}}{\Gamma(\alpha+k-m+1) \varrho^{\alpha-m+k}} \\
& \times\left({ }_{\ominus} D^{k, \varrho, g} f\right)(b) .
\end{aligned}
$$

Proof The proof can be done by mathematical induction.

In the following theorems we present the impact of the fractional integral on the fractional derivative of the same order.

Theorem 3.1 Let $\operatorname{Re}(\alpha)>0, n=-[-\operatorname{Re}(\alpha)], f \in L_{1}(a, b)$, and $\left({ }_{a}{ }^{\alpha, \rho, g} f\right)(t),\left(I_{b}^{\alpha, \rho, g} f\right)(t) \in$ $A C^{n}[a, b]$. Then

$$
\begin{aligned}
\left({ }_{a} I^{\alpha, \varrho, g}{ }_{a} D^{\alpha, \varrho, g} f(t)=\right. & f(t) \\
& -e^{\frac{\varrho-1}{\varrho}(g(t)-g(a))} \sum_{j=1}^{n}\left({ }_{a} I^{j-\alpha, \varrho, g} f\right)\left(a^{+}\right) \frac{(g(t)-g(a))^{\alpha-j}}{\varrho^{\alpha-j} \Gamma(\alpha+1-j)}
\end{aligned}
$$

and

$$
\begin{aligned}
\left(I_{b}^{\alpha, \varrho, g}{ }_{\ominus} D_{b}^{\alpha, \varrho, g} f(t)=\right. & f(t) \\
& -e^{\frac{\varrho-1}{\varrho}(g(b)-g(t))} \sum_{j=1}^{n}(-1)^{j}\left(I_{b}^{j-\alpha, \varrho, g} f\right)\left(b^{-}\right) \frac{(g(b)-g(t))^{\alpha-j}}{\varrho^{\alpha-j} \Gamma(\alpha+1-j)} .
\end{aligned}
$$

Proof By applying Corollary 3.1 and Theorem 2.1, we can observe that

$$
\begin{aligned}
{ }_{a} I^{\alpha, \varrho}{ }_{a} D^{\alpha, \varrho} f(t)= & { }_{a} I^{\alpha, \varrho} D^{n, \varrho}{ }_{a} I^{n-\alpha, \varrho} f(t) \\
= & D^{n, \varrho}{ }_{a} I^{\alpha, \varrho}{ }_{a} I^{n-\alpha, \varrho} f(t) \\
& -\sum_{k=0}^{n-1} \frac{(t-a)^{\alpha-n+k} e^{\frac{\varrho-1}{\varrho}(t-a)}}{\Gamma(\alpha+k-n+1) \varrho^{\alpha-n+k}}\left(D^{k, \varrho}{ }_{a} I^{n-\alpha, \varrho} f\right)(a) \\
= & f(t)-\sum_{k=0}^{n-1} \frac{(t-a)^{\alpha-n+k} e^{\frac{\varrho-1}{\varrho}(t-a)}}{\Gamma(\alpha+k-n+1) \varrho^{\alpha-n+k}}\left({ }_{a} I^{n-\alpha-k, \varrho} f\right)\left(a^{+}\right) \\
= & f(t)-e^{\frac{\varrho-1}{\varrho}(t-a)} \sum_{j=1}^{n}\left({ }_{a} I^{j-\alpha, \varrho} f\right)\left(a^{+}\right) \frac{(t-a)^{\alpha-j}}{\varrho^{\alpha-j} \Gamma(\alpha+1-j)},
\end{aligned}
$$

where the change of variable $j=n-k$ has been used. Equation (3.6) can be analogously proved.

Theorem 3.2 For $\varrho>0$ and $n=[\operatorname{Re}(\alpha)]+1$, we have

$$
{ }_{a} I^{\alpha, \varrho, g}\left({ }_{a}^{C} D^{\alpha, \varrho, g} f\right)(t)=f(t)-\sum_{k=0}^{n-1} \frac{\left(D^{k, \varrho, g} f\right)(a)}{\varrho^{k} k !}(g(t)-g(a))^{k} e^{\frac{\varrho-1}{\varrho}(g(t)-g(a))}
$$


and

$$
I_{b}^{\alpha, \varrho, g}\left({ }^{C} D_{b}^{\alpha, \varrho, g} f\right)(t)=f(t)-\sum_{k=0}^{n-1} \frac{(-1)^{k}\left({ }_{\ominus} D^{k, \varrho, g} f\right)(b)}{\varrho^{k} k !}(g(b)-g(t))^{k} e^{\frac{\varrho-1}{\varrho}(g(b)-g(t))} .
$$

Proof By the help of Theorem 3.1 with $\alpha=n$, we have

$$
\begin{aligned}
{ }_{a} I^{\alpha, \varrho, g}\left({ }_{a}^{C} D^{\alpha, \varrho, g} f\right)(t)= & { }_{a} I^{\alpha, \varrho, g}\left({ }_{a} I^{n-\alpha, \varrho, g} D^{n, \varrho, g} f\right)(t)=\left({ }_{a} I^{n, \varrho, g} D^{n, \varrho, g} f\right)(t) \\
= & f(t) \\
& -e^{\frac{\varrho-1}{\varrho}(g(t)-g(a))} \sum_{j=1}^{n} \frac{\left({ }_{a} I^{j-n, \varrho, g} f\right)\left(a^{+}\right)(g(t)-g(a))^{n-j}}{\varrho^{n-j} \Gamma(n-j+1)} \\
= & f(t)-\sum_{k=0}^{n-1} \frac{\left(D^{k, \varrho, g} f\right)(a)}{\varrho^{k} k !}(g(t)-g(a))^{k} e^{\frac{\varrho-1}{\varrho}(g(t)-g(a))} .
\end{aligned}
$$

Equation (3.8) can be proved similarly.

Before we find the $g$-Laplace transform, we need to find the $g$-Laplace transform of the $n^{t} h$ order derivative of a function with respect to another function.

Theorem 3.3 Let the function $f \in C_{g}[a, T]$ and of $g$-exponential order such that $D^{\varrho, g} f$ is piecewise continuous over every finite interval $[a, T]$. Then the generalized Laplace transform of $D^{\varrho, g} f$ exists and

$$
\mathcal{L}_{g}\left\{D^{\varrho, g} f(t)\right\}(s)=(\varrho s+1-\varrho) \mathcal{L}_{g}\{f(t)\}(s)-\varrho f(a) .
$$

Proof Using (2.10) and Theorem 2.3, we have

$$
\begin{aligned}
\mathcal{L}_{g}\left\{D^{\varrho, g} f(t)\right\}(s) & =(1-\varrho) \mathcal{L}_{g}\{f(t)\}+\varrho \mathcal{L}_{g}\left\{f^{[1]}(t)\right\}(s) \\
& =(1-\varrho) \mathcal{L}_{g}\{f(t)\}+\varrho\left(s \mathcal{L}_{g}\{f(t)\}(s)-f(a)\right) \\
& =(\varrho s+1-\varrho) \mathcal{L}_{g}\{f(t)\}(s)-\varrho f(a) .
\end{aligned}
$$

Using induction on $n$, we can prove the following corollary.

Corollary 3.2 Let $f \in C^{n-1}[a, \infty)$ be such that $f^{[i]}, i=1,2, \ldots, n-1$, are of exponential order $e^{c t}$ on each subinterval $[a, T]$. Then

$$
\begin{aligned}
\mathcal{L}_{g}\left\{\left(D^{n, \varrho, g} f\right)(t)\right\}(s)= & (\varrho s+1-\varrho)^{n} \mathcal{L}_{g}\{f(t)\}(s) \\
& -\varrho \sum_{k=0}^{n-1}(\varrho s+1-\varrho)^{n-1-k}\left(D^{k, \varrho, g} f\right)(a) .
\end{aligned}
$$

Theorem 3.4 Let $\alpha \in \mathbb{C}$ with $\operatorname{Re}(\alpha)>0$ and $\varrho \in(0],, n=[\operatorname{Re}(\alpha)]+1$. Assume that $f$ is of g-exponential order. Then

$$
\mathcal{L}_{g}\left\{\left({ }_{a} I^{\alpha, \varrho, g} f\right)(t)\right\}(s)=\frac{1}{(\varrho s+1-\varrho)^{\alpha}} \mathcal{L}_{g}\{f(t)\}(s), \quad s>c .
$$


Proof From the convolution formula we have

$$
\begin{aligned}
\mathcal{L}_{g}\left\{\left({ }_{a} I^{\alpha, \varrho, g} f\right)(t)\right\}(s) & =\frac{1}{\varrho^{\alpha} \Gamma(\alpha)} \mathcal{L}_{g}\left\{e^{\frac{\varrho-1}{\varrho}(g(t)-g(a))}(g(t)-g(a))^{\alpha-1} *_{g} f(t)\right\}(s) \\
& =\frac{1}{\varrho^{\alpha} \Gamma(\alpha)} \frac{\Gamma(\alpha)}{\left(s-\frac{\varrho-1}{\varrho}\right)^{\alpha}} \mathcal{L}_{a}\{f(t)\}(s) \\
& =\frac{1}{(\varrho s+1-\varrho)^{\alpha}} \mathcal{L}_{a}\{f(t)\}(s) .
\end{aligned}
$$

Theorem 3.5 For any $\alpha \in \mathbb{C}$ with $\operatorname{Re}(\alpha)>0$ and $\varrho \in(0,1], n=[\operatorname{Re}(\alpha)]+1$, we have

$$
\begin{aligned}
\mathcal{L}_{g}\left\{\left({ }_{a} D^{\alpha, \varrho, g} f\right)(t)\right\}(s)= & (\varrho s+1-\varrho)^{\alpha} F_{g}(s) \\
& -\varrho \sum_{k=0}^{n-1}(\varrho s+1-\varrho)^{n-k-1}\left(I^{n-\alpha-k, \varrho, g} f\right)\left(a^{+}\right)
\end{aligned}
$$

where $F_{g}(s)=\mathcal{L}_{g}\{f(t)\}(s)$. In particular, iff is continuous at a, then

$$
\mathcal{L}_{g}\left\{\left({ }_{a} D^{\alpha, \varrho, g} f\right)(t)\right\}(s)=(\varrho s+1-\varrho)^{\alpha} F_{g}(s) .
$$

Proof By applying Corollary 3.2 and Theorem 3.4, we have

$$
\begin{aligned}
\mathcal{L}_{a}\left\{\left({ }_{a} D^{\alpha, \varrho} f\right)(t)\right\}(s)= & \left.\mathcal{L}_{g}\left\{{ }_{a} D^{n, \varrho, g}{ }_{a} I^{n-\alpha, \varrho, g} f\right)(t)\right\}(s) \\
= & \left.(\varrho s-1+\varrho)^{n} \mathcal{L}_{g}\left\{{ }_{a} I^{n-\alpha, \varrho, g} f\right)(t)\right\}(s) \\
& -\varrho \sum_{k=0}^{n-1}(\varrho s-1+\varrho)^{n-1-k}\left(D^{k, \varrho, g}{ }_{a} I^{n-\alpha, \varrho, g} f\right)\left(a^{+}\right) \\
= & (\varrho s+1-\varrho)^{n}(\varrho s+1-\varrho)^{\alpha-n} F_{g}(s) \\
& -\varrho \sum_{k=0}^{n-1}(\varrho s-1+\varrho)^{n-1-k}\left({ }_{a} I^{n-\alpha-k, \varrho, g} f\right)\left(a^{+}\right) .
\end{aligned}
$$

The last part follows by noting that $\left({ }_{a} I^{n-\alpha-k, \varrho} f\right)\left(a^{+}\right)$vanishes for a continuous function $f$ on $[a, b]$.

Theorem 3.6 Let $\alpha \in \mathbb{C}$ with $\operatorname{Re}(\alpha)>0$ and $\varrho>0, n=[\operatorname{Re}(\alpha)]+1$. If $F_{g}(s)=\mathcal{L}\{f(t)\}(s)$, then

$$
\mathcal{L}_{a}\left\{\left({ }_{a}^{C} D^{\alpha, \varrho} f\right)(t)\right\}(s)=(\varrho s+1-\varrho)^{\alpha} F_{a}(s)-\varrho \sum_{k=0}^{n-1}(\varrho s+1-\varrho)^{\alpha-1-k}\left(D^{k, \varrho} f\right)(a) .
$$

Proof

$$
\begin{aligned}
\mathcal{L}_{g}\left\{\left({ }_{a}^{C} D^{\alpha, \varrho, g} f\right)(t)\right\}(s)= & \mathcal{L}_{g}\left\{\left({ }_{a} I^{n-\alpha, \varrho, g} D^{n, \varrho, g} f\right)(t)\right\}(s) \\
= & (\varrho s+1-\varrho)^{\alpha-n} \mathcal{L}_{g}\left\{\left(D^{\alpha, \varrho, g} f\right)(t)\right\}(s) \\
= & (\varrho s+1-\varrho)^{\alpha-n} \\
& \times\left[(\varrho s+1-\varrho)^{n} F_{g}(s)\right.
\end{aligned}
$$




$$
\begin{aligned}
& \left.-\varrho \sum_{k=0}^{n-1}(\varrho s+1-\varrho)^{n-1-k}\left(D^{k, \varrho, g} f\right)(a)\right] \\
= & (\varrho s+1-\varrho)^{\alpha} F_{g}(s) \\
& -\varrho \sum_{k=0}^{n-1}(\varrho s+1-\varrho)^{\alpha-1-k}\left(D^{k, \varrho, g} f\right)(a) .
\end{aligned}
$$

Using the Laplace transform of the generalized proportional derivatives in RiemannLiouville settings in Theorem 3.5 and the Laplace transform in the Caputo in Theorem 3.6, we can state the following relation that links the Caputo and Riemann-Liouville general proportional fractional derivatives.

Corollary 3.3 For any $\alpha \in \mathbb{C}$ with $\operatorname{Re}(\alpha)>0$ and $\varrho>0, n=[\operatorname{Re}(\alpha)]+1$, we have

$$
\begin{aligned}
\left({ }_{a}^{C} D^{\alpha, \varrho} f\right)(t)= & \left({ }_{a} D^{\alpha, \varrho, g} f\right)(t) \\
& -\sum_{k=0}^{n-1} \frac{\varrho^{\alpha-k}}{\Gamma(k+1-\alpha)}(g(t)-g(a))^{k-\alpha} e^{\frac{\varrho-1}{\varrho}(g(t)-g(a))}\left(D^{k, \varrho, g} f\right)(a) .
\end{aligned}
$$

Example 3.1 Consider the linear proportional fractional initial value problem

$$
{ }_{a} D^{\alpha, \varrho, g} y(t)-\varrho^{\alpha} \lambda y(t)=f(t), \quad\left({ }_{a} I^{1-\alpha, \varrho, g} y\right)\left(a^{+}\right)=y_{a}, \quad 0<\alpha \leq 1 .
$$

Then $y(t)$ is a solution of (3.15) if and only if it satisfies the integral equation

$$
\begin{aligned}
y(t)= & y_{a} \varrho^{1-\alpha} e^{\frac{\varrho-1}{\varrho}(g(t)-g(a))}(g(t)-g(a))^{\alpha-1} E_{\alpha, \alpha}\left(\lambda(g(t)-g(a))^{\alpha}\right) \\
& +\varrho^{-\alpha} \int_{a}^{t} E_{\alpha, \alpha}\left(\lambda(g(t)-g(s))^{\alpha}\right) e^{\frac{\varrho-1}{\varrho}(g(t)-g(s))}(g(t)-g(s))^{\alpha-1} \\
& \times f(s) g^{\prime}(s) d s,
\end{aligned}
$$

where $E_{\alpha, \beta}$ is the Mittag-Leffler function of two parameters [29]. Actually, applying $\mathcal{L}_{g}$ to (3.15) and making use of Theorem 3.5 with $n=1$, we obtain

$$
\left((\varrho s+1-\varrho)^{\alpha}-\lambda \varrho^{\alpha}\right) Y_{g}(s)=\varrho y_{a}+F_{a}(s)
$$

Hence,

$$
Y_{a}(s)=\frac{\varrho^{1-\alpha} y_{a}}{\left(s-\frac{\varrho-1}{\varrho}\right)^{\alpha}-\lambda}+\frac{\varrho^{-\alpha} F_{g}(s)}{\left(s-\frac{\varrho-1}{\varrho}\right)^{\alpha}-\lambda} .
$$

Applying the inverse of $\mathcal{L}_{a}$ and using the fact that [21]

$$
\mathcal{L}_{g}\left\{(g(t)-g(a))^{\alpha-1} E_{\alpha, \alpha}\left(\lambda(g(t)-g(a))^{\alpha}\right)\right\}=\frac{1}{s^{\alpha}-\lambda},
$$

together with Theorem 2.4, we reach (3.16). Conversely, if $y(t)$ has the representation (3.16), then by the help of Proposition 2.1 it satisfies (3.15). 
Example 3.2 Consider the linear Caputo proportional fractional initial value problem

$$
{ }_{a}^{C} D^{\alpha, \varrho, g} y(t)-\varrho^{\alpha} \lambda y(t)=f(t), \quad y(a)=y_{a}, 0<\alpha \leq 1 .
$$

Then $y(t)$ is a solution of (3.17) if and only if it satisfies the integral equation

$$
\begin{aligned}
y(t)= & y_{a} e^{\frac{\varrho-1}{\varrho}(g(t)-g(a))}(g(t)-g(a))^{\alpha-1} E_{\alpha}\left(\lambda(g(t)-g(a))^{\alpha}\right) \\
& +\varrho^{-\alpha} \int_{a}^{t} E_{\alpha, \alpha}\left(\lambda(g(t)-g(s))^{\alpha}\right) e^{\frac{\varrho-1}{\varrho}(g(t)-g(s))}(g(t)-g(s))^{\alpha-1} \\
& \times f(s) g^{\prime}(s) d s,
\end{aligned}
$$

where $E_{\alpha}$ is the Mittag-Leffler function of one parameter [29]. Applying $\mathcal{L}_{a}$ to (3.17) and making use of Theorem 3.6 with $n=1$, we have

$$
\left((\varrho s+1-\varrho)^{\alpha}-\lambda \varrho^{\alpha}\right) Y_{g}(s)=\varrho y_{a}(\varrho s+1-\varrho)^{\alpha-1}+F_{g}(s)
$$

Hence,

$$
Y_{g}(s)=\frac{\left(s-\frac{\varrho-1}{\varrho}\right)^{\alpha-1} y_{a}}{\left(s-\frac{\varrho-1}{\varrho}\right)^{\alpha}-\lambda}+\frac{\varrho^{-\alpha} F_{g}(s)}{\left(\frac{\varrho-1}{\varrho}-s\right)^{\alpha}-\lambda} .
$$

Applying the inverse of $\mathcal{L}_{a}$ and using the facts that

$$
\mathcal{L}_{g}\left\{\left(g((t)-g(a))^{\alpha-1} E_{\alpha, \alpha}\left(\lambda(g(t)-g(a))^{\alpha}\right)\right\}=\frac{1}{s^{\alpha}-\lambda}\right.
$$

and

$$
\mathcal{L}_{g}\left\{E_{\alpha}\left(\lambda(g(t)-g(a))^{\alpha}\right)\right\}=\frac{s^{\alpha-1}}{s^{\alpha}-\lambda}
$$

together with the convolution formula, we reach (3.18). Conversely, if $y(t)$ has the representation (3.18), then by the help of Proposition 2.2 one can easily show that it satisfies (3.17).

\section{Some integration by parts formulas}

In this section, we present some integration by parts formulas.

Theorem 4.1 Letf, $h, g \in C^{n}[a, b], g^{\prime}(t)>0, \alpha \in \mathbb{R}, n=[\alpha]+1$, and $\varrho \in(0,1]$. Then

$$
\begin{aligned}
\int_{a}^{b} f(t)\left({ }_{a}^{C} D^{\alpha, \varrho, g} h\right)(t) g^{\prime}(t) d t= & \int_{a}^{b} h(t)\left({ }^{C} D_{b}^{\alpha, e, g} f\right)(t) g^{\prime}(t) d t \\
& +\left.\varrho\left[\sum_{k=0}^{n-1}\left(D^{k, e, g} h\right)(t)\left(I_{b}^{k-\alpha, \varrho, g} f\right)(t)\right]\right|_{a} ^{b}
\end{aligned}
$$

Proof

$$
\int_{a}^{b} f(t)\left({ }_{a}^{C} D^{\alpha, \varrho, g} h\right)(t) g^{\prime}(t) d t
$$




$$
\begin{aligned}
= & \int_{a}^{b} f(t) \frac{1}{\varrho^{n-\alpha} \Gamma(n-\alpha)} \int_{a}^{t} e^{\frac{\varrho-1}{\varrho}(g(t)-g(s))}(g(t)-g(s))^{n-\alpha-1} \\
& \times\left(D^{n, \varrho, g} h\right)(s) g^{\prime}(s) d s g^{\prime}(t) d t \\
= & \int_{a}^{b}\left(D^{n, \varrho, g} h\right)(s) \frac{1}{\varrho^{n-\alpha} \Gamma(n-\alpha)} \int_{s}^{b} e^{\frac{\varrho-1}{\varrho}(g(t)-g(s))}(g(t)-g(s))^{n-\alpha-1} \\
& \times f(t) g^{\prime}(t) d t g^{\prime}(s) d s \\
= & \int_{a}^{b}\left(D^{n, \varrho, g} h\right)(s)\left(I_{b}^{\alpha, \varrho, g} f\right)(s) g^{\prime}(s) d s \\
= & \int_{a}^{b}(1-\varrho)\left(D^{n-1, \varrho, g} h\right)(s)\left(I_{b}^{\alpha, \varrho, g} f\right)(s) g^{\prime}(s) \\
& +\varrho\left(\frac{d}{d s}\left(D^{n-1, \varrho, g} h\right)\right)(s)\left(I_{b}^{\alpha, \varrho, g} f(s) d s\right. \\
= & \int_{a}^{b}(1-\varrho)\left(D^{n-1, \varrho, g} h\right)(s) g^{\prime}(s)-\varrho\left(D^{n-1, \varrho, g} h\right)(s) \frac{d}{d s}\left(\left(I_{b}^{\alpha, \varrho, g} f\right)(s)\right) \\
& +\left.\varrho\left[\left(D^{n-1, \varrho, g} h\right)(s)\left(I_{b}^{\alpha, \varrho, g} f\right)(s)\right]\right|_{a} ^{b} .
\end{aligned}
$$

The result is obtained by repeating the above procedure $n-1$ times.

Using Theorem 4.1 and the relation between the Caputo and Riemann-Liouville type derivatives, we can conclude the following.

\section{Corollary 4.1}

$$
\begin{aligned}
\int_{a}^{b} f(t)\left({ }_{a} D^{\alpha, \varrho, g} h\right)(t) g^{\prime}(t) d t= & \int_{a}^{b} h(t)\left(D_{b}^{\alpha, \varrho, g} f\right)(t) g^{\prime}(t) d t \\
& +\varrho \sum_{k=0}^{n-1}\left(D^{k, \varrho, g} h\right)(b)\left(I_{b}^{k+1-\alpha, \varrho, g} f\right)(b) .
\end{aligned}
$$

Analogously, the following theorem and corollary can be proved.

Theorem 4.2 Letf, $h, g \in C^{n}[a, b], g^{\prime}(t)>0, \alpha \in \mathbb{R}, n=[\alpha]+1$, and $\varrho \in(0,1]$. Then

$$
\begin{aligned}
\int_{a}^{b} f(t)\left({ }^{C} D_{b}^{\alpha, \varrho, g} h\right)(t) g^{\prime}(t) d t= & \int_{a}^{b} h(t)\left({ }_{a} D^{\alpha, \varrho, g} f\right)(t) g^{\prime}(t) d t \\
& -\left.\varrho\left[\sum_{k=0}^{n-1}\left(D^{k, \varrho, g} h\right)(t)\left({ }_{a} I^{k-\alpha, \varrho, g} f\right)(t)\right]\right|_{a} ^{b}
\end{aligned}
$$

\section{Corollary 4.2}

$$
\begin{aligned}
\int_{a}^{b} f(t)\left(D_{b}^{\alpha, \varrho, g} h\right)(t) g^{\prime}(t) d t= & \int_{a}^{b} h(t)\left({ }_{a} D^{\alpha, \varrho, g} f\right)(t) g^{\prime}(t) d t \\
& +\varrho \sum_{k=0}^{n-1}\left({ }_{\ominus} D^{k, \varrho, g} h\right)(a)\left({ }_{a} I^{k-\alpha+1, \varrho, g} f\right)(a) .
\end{aligned}
$$




\section{Conclusions}

We presented the activities of the general fractional proportional integrals on the general fractional proportional derivatives. These actions are indispensable for the study of the qualitative aspects of differential and integral equations in the frame of the considered operators. In addition, we heralded the suitable integral transforms of these operators that we believe will help in discussing the stability of systems involving such fractional operators.

The proportional derivatives $D^{\varrho}$ and the proportional derivative are defined only when $0 \leq \varrho \leq 1$, and higher-order derivatives are defined when we have a sequential of these derivatives. This may cause some obstacles in dealing with equations containing such derivatives. For this reason, we present the following definition of the proportional derivative of any order $n \leq \varrho \leq n+1, n=0,1, \ldots$ :

$$
D^{\varrho} f(t)=(n+1-\varrho) f^{(n)}(t)+(\varrho-n) f^{(n+1)}(t)
$$

which is equivalent to Definition 5.5 in [30]. It can be clearly observed that this derivative is an interpolation between the $n$th derivative of the function and its derivative of order $n+1$. We believe that it would be interesting to work on such a definition.

\section{Acknowledgements}

The second author would like to thank Prince Sultan University for funding this work through research group Nonlinear Analysis Methods in Applied Mathematics (NAMAM) group number RG-DES-2017-01-17.

\section{Funding}

Not applicable.

Availability of data and materials

Not applicable.

\section{Competing interests}

The authors declare that they have no competing interests.

\section{Authors' contributions}

All authors contributed equally to this article. All authors read and approved the final manuscript.

\section{Author details}

${ }^{1}$ Department of Mathematics, Çankaya University, Ankara, Turkey. ${ }^{2}$ Department of Mathematics and General Sciences, Prince Sultan University, Riyadh, Saudi Arabia. ${ }^{3}$ Department of Medical Research, China Medical University, Taichung, Taiwan. ${ }^{4}$ Department of Computer Science and Information Engineering, Asia University, Taichung, Taiwan.

${ }^{5}$ Department of Mathematics, Government College University, Faisalabad, Pakistan. ${ }^{6}$ Equipe E3MI, Department of Mathematics, FST Errachidia, University Moulay Ismail, Errachidia, Morocco.

\section{Publisher's Note}

Springer Nature remains neutral with regard to jurisdictional claims in published maps and institutional affiliations.

Received: 4 May 2020 Accepted: 10 June 2020 Published online: 19 June 2020

\section{References}

1. Hilfer, R:: Applications of Fractional Calculus in Physics. World Scientific, Singapore (2000)

2. Debnath, L.: Recent applications of fractional calculus to science and engineering. Int. J. Math. Math. Sci. 54, 3413-3442 (2003)

3. Samko, S.G., Kilbas, A.A., Marichev, O.I.: Fractional Integrals and Derivatives: Theory and Applications. Gordon \& Breach, Yverdon (1993)

4. Kilbas, A., Srivastava, H.M., Trujillo, J.J.: Theory and Application of Fractional Differential Equations. North-Holland Mathematics Studies, vol. 204. Elsevier, Amsterdam (2006)

5. Magin, R.L.: Fractional Calculus in Bioengineering. Begell House Publishers (2006)

6. Podlubny, I.: Fractional Differential Equations. Academic Press, San Diego (1999)

7. Caputo, M., Fabrizio, M.: A new definition of fractional derivative without singular kernel. Prog. Fract. Differ. Appl. 1, 73 (2015) 
8. Atangana, A., Baleanu, D.: New fractional derivative with non-local and non-singular kernel. Therm. Sci. 20, 757 (2016)

9. Jarad, F., Abdeljawad, T., Hammouch, Z:: On a class of ordinary differential equations in the frame of Atangana-Baleanu fractional derivative. Chaos Solitons Fractals 117, 16-20 (2018)

10. Shah, K., Jarad, F., Abdeljawad, T.: On a nonlinear fractional order model of dengue fever disease under Caputo-Fabrizio derivative. Alex. Eng. J. https://doi.org/10.1016/j.aej.2020.02.022

11. Shah, K., Alqudah, M.A., Jarad, F., Abdeljawad, T.: Semi-analytical study of pine wilt disease model with convex rate under Caputo-Fabrizio fractional order derivative. Chaos Solitons Fractals 135, 109754 (2020)

12. Nazir, G., Shah, K., Alrabaiah, H., Khalil, H., Khan, R.A.: Fractional dynamical analysis of measles spread model under vaccination corresponding to nonsingular fractional order derivative. Adv. Differ. Equ. 2020, 171 (2020)

13. Yavuz, M., Özdemir, N.: Comparing the new fractional derivative operators involving exponential and Mittag-Leffler kernel. Discrete Contin. Dyn. Syst. 13(3), 995-1006 (2020)

14. Yavuz, M., Özdemir, N.: European vanilla option pricing model of fractional order without singular kernel. Fractal Fract. 2(1), 3 (2018)

15. Katugampola, U.N.: New approach to generalized fractional integral. Appl. Math. Comput. 218, 860-865 (2011)

16. Katugampola, U.N.: A new approach to generalized fractional derivatives. Bull. Math. Anal. Appl. 6, 1-15 (2014)

17. Jarad, F., Abdeljawad, T., Baleanu, D.: On the generalized fractional derivatives and their Caputo modification. J. Nonlinear Sci. Appl. 10(5), 2607-2619 (2017)

18. Khalil, R., Al Horani, M., Yousef, A., Sababheh, M.: A new definition of fractional derivative. J. Comput. Appl. Math. 264, 65-70 (2014)

19. Abdeljawad, T.: On conformable fractional calculus. J. Comput. Appl. Math. 279, 57-66 (2013)

20. Jarad, F., Ugurlu, E., Abdeljawad, T., Baleanu, D.: On a new class of fractional operators. Adv. Differ. Equ. 2018, 142 (2018)

21. Jarad, F., Abdeljawad, T.: Generalized fractional derivatives and Laplace transform. Discrete Contin. Dyn. Syst., Ser. S 13(3), 709-722 (2020)

22. Anderson, D.R., Ulness, D.J.: Newly defined conformable derivatives. Adv. Dyn. Syst. Appl. 10(2), 109-137 (2015)

23. Anderson, D.R.: Second-order self-adjoint differential equations using a proportional-derivative controller. Commun. Appl. Nonlinear Anal. 24, 17-48 (2017)

24. Jarad, F., Abdeljawad, T., Alzabut, J.: Generalized fractional derivatives generated by a class of local proportional derivatives. Eur. Phys. J. Spec. Top. 226, 3457-3471 (2017)

25. Jarad, F., Alqudah, M.A., Abdeljawad, T.: On more generalized form of proportional fractional operators. Open Math. $18,167-176(2020)$

26. Rashid, S., Jarad, F., Noor, M.A., Kalsoom, H.: Inequalities by means of generalized proportional fractional integral operators with respect to another function. Mathematics 7(12), 1225 (2019). https://doi.org/10.3390/math7121225

27. Rahman, G., Abdeljawad, T., Jarad, F., Khan, A., Nisar, K.S.: Bounds of generalized proportional fractional integrals in general form via convex functions and their applications. Mathematics 8(1), 113 (2020). https://doi.org/10.3390/math8010113

28. Rahman, G., Abdeljawad, T., Jarad, F., Khan, A., Nisar, K.S.: Certain inequalities via generalized proportional Hadamard fractional integral operators. Adv. Differ. Equ. 2019, 454 (2019)

29. Kilbas, A.A., Saigo, M., Saxena, K.: Generalized Mittag-Leffler function and generalized fractional calculus operators Integral Transforms Spec. Funct. 15, 31-49 (2004)

30. Abdeljawad, T.: Lyapunov-type inequalities for local fractional proportional derivatives. In: Dutta, H., Akdemir, A.O., Atangana, A. (eds.) Fractional Order Analysis: Theory, Methods and Applications. Wiley, New York (2020)

\section{Submit your manuscript to a SpringerOpen ${ }^{\circ}$ journal and benefit from:}

- Convenient online submission

- Rigorous peer review

- Open access: articles freely available online

- High visibility within the field

- Retaining the copyright to your article

Submit your next manuscript at $\boldsymbol{~ s p r i n g e r o p e n . c o m ~}$ 